\title{
Emerging contaminants and nanoplastics in the water environment: a matter of rising concern
}

\author{
Antonio Ginebreda \\ Environmental Chemistry, IDAEA-CSIC, Barcelona 08034, Spain. \\ Correspondence to: Prof. Antonio Ginebreda, Environmental Chemistry, IDAEA-CSIC, c/ Jordi Girona 18-26, Barcelona 08034, \\ Spain. E-mail: agmqam@cid.csic.es
}

How to cite this article: Ginebreda A. Emerging contaminants and nanoplastics in the water environment: a matter of rising concern. Water Emerg Contam Nanoplastics 2021;1:1. https://dx.doi.org/10.20517/wecn.2021.02

Received: 2 Dec 2021 Accepted: 13 Dec 2021 Published: 20 Dec 2021

Academic Editor: Teresa A. P. Rocha-Santos Copy Editor: Xi-Jun Chen Production Editor: Xi-Jun Chen

\section{INTRODUCTION}

Our highly technological society is strongly based on the extensive use of chemicals. About 140,000 chemicals are currently on the market and can be thus considered of common use by industry and households ${ }^{[1,2]}$, however, the number of available chemicals largely exceeds this quantity and is continuously growing. Depending on both natural (physical-chemical properties or environmental physical conditions) and anthropogenic factors (volumes produced, mode of use, and waste treatment technologies). these chemicals may potentially reach the environment. As prescribed by the Second Principle of Thermodynamics. even the best environmental practices such as waste treatment, reuse, and recycling cannot avoid certain losses to the environment in the form of thermal and material entropy ${ }^{[3]}$. This is particularly true for those compounds, such as pesticides, whose mode of use consists of openenvironmental application.

Progress on environment pollution knowledge is tightly coupled to that of the analytical methods. In the field of organic micropollutants, these are largely based on the joint evolution of separation techniques, notably gas and liquid chromatography (GC and LC, respectively), and powerful identification methods such as mass spectrometry (MS). Detection levels of nanograms or picograms are nowadays routinely reached with the currently available instruments. Continuous lowering of detection limits 
Table 1. Environmental concerns associated with emerging contaminants (EC) and their transformation products

- Chemicals are regularly used by the population and the industry with a clear growing trend. The process is ubiquitous in space and continuous along time and extends to some hundreds of thousands of available compounds

- Water treatment systems (if any) are not specifically designed to eliminate many ECs. Depending on each specific compound and characteristics of the treatment, their elimination might not be complete. Further tertiary specific treatment steps may be necessary, but at a certain economic cost that is not always affordable

- Even though many ECs may be degraded, their continuous input into the environment makes them behave as "pseudo-persistent"

- They are often polar compounds, which are difficult to extract and analyze

- The complex "cocktail" of many ECs, metabolites, and transformation products may have unexpected and hardly predictable mixture interaction effects (i.e., synergistic, antagonistic, etc.)

- Legislation is limited to a list of a few compounds compared to the large number potentially present in the environment. Mixture effects are overlooked

- Some ECs are, by design, biologically active compounds (i.e., pesticides, pharmaceuticals, etc.), targeted to specific organisms and having precise modes of action. Possible side effects on other non-targeted, unintentionally exposed organisms found in the receiving ecosystem biological communities are largely unknown

- Finally, the anthropogenic water cycle includes the safe supply of drinking water to the population. The occurrence of EC residues in drinking water may pose a risk to human health, therefore their absence must be prevented in any case

(by a factor of ca. $10^{6}$ in the last 40 years) has led to the "discovery" of more and more contaminants in the environment that otherwise were overlooked, although they were obviously already present. These contaminants have remained ignored as well by the legislation since they (apparently) did not meet the requirements of "persistence, bioaccumulation, and toxicity (PBT)". However, their continuous release to the environment can qualify them as "pseudo-persistent", their biological activity by design in some cases (i.e., pesticides, pharmaceuticals, etc.), their unknown effects resulting from the continuous and simultaneous exposure to low doses of many of such contaminants, or the discovery of specific biological effects associated (i.e., endocrine disruption) have been recognized as a relevant environmental threat, which has led to coining the term "contaminants of emerging concern", or in short "emerging contaminants" [Table 1]. Nowadays, under this broad definition, various families of contaminants are allocated (i.e., pesticides, pharmaceuticals, personal care products, endocrine disruptors, perfluorinated compounds, flame retardants, UV filters, etc.). This is a dynamic definition, and the list remains open to the continuous incorporations of more candidates resulting from new findings.

Coping with chemical pollution is therefore of utmost relevance. Focusing on the anthropogenic water cycle, the situation is particularly worrying if one considers that about $80 \%$ of the world's wastewaters lack any treatment, but even conventional processes are not able to remove many pollutants and further advanced treatments are required ${ }^{[4]}$. On the other hand, the current legislation has a limited scope, typically setting environmental quality standard thresholds levels for a limited number of compounds and overlooking any mixture effects ${ }^{[5]}$.

\section{POLLUTANTS IN THE WATER ENVIRONMENT: BRIDGING THE GAP BETWEEN CHEMISTRY AND BIOLOGY}

Once contaminants reach the aquatic environment, they may undergo biotic (i.e., biodegradation) and abiotic transformations (hydrolysis, photolysis, redox processes, etc.) that give rise to a plethora of environmentally generated by-products altogether broadly known as "transformation products (TPs)". It should be emphasized that the term "aquatic environment" must be understood in its broadest sense, covering both natural (surface freshwaters, groundwaters, transitional, and coastal waters) and engineered systems (potabilization and wastewater treatment), as well as different environmental compartments (water phase, sediments, suspended solids, and biota). 
Parent compounds plus the resulting TPs constitute a "chemical universe" (chemosphere) whose interactions with the different levels of biological organization, i.e., species, individuals, populations, and ecosystems (ecosphere), are still poorly known, but many experimental pieces of evidence show that they may result on harmful effects ${ }^{[0,7]}$. The interrelation between chemical exposure and their potential adverse biological effects at different levels of biological complexity (including humans) compose altogether a chain of events known in the current environmental toxicology literature as adverse outcome pathways whose investigation appears to be crucial ${ }^{[8,9]}$.

\section{Chemical status vs. ecological status}

The relationships between chemical and ecological status may be regarded as one of the ultimate goals to be achieved in our understanding of chemical pollution consequences ${ }^{[10]}$. Several factors complicate the overall picture. First, chemical stressors entering watercourses co-occur in mixtures of many different compounds, making the prediction of their joint effects more difficult ${ }^{[6]}$. Second, these organic microcontaminants may coincide with nutrients in excess or with abundant dissolved organic matter, especially in systems heavily impacted by industrial or urban effluents, making up a number of co-occurring stressors with complex effects on the biological communities that may confound the resulting outcome $\mathrm{e}^{[1]}$. Finally, the effects of these stressors on the biological compartments (i.e., from bacteria to fish) are often non-linear, and outputs become increasingly uncertain through larger spatial and temporal scales of complexity in which other factors such as hydrological, hydromorphological, climate (temperature), and land-use changes play their role as well ${ }^{[1,12]}$. Disentanglement of these complex scenarios remains a challenging task. Some approaches aim to allocate the contribution of different co-occurring stressors based on variance partitioning ${ }^{[13]}$, study of macroinvertebrate traits ${ }^{[14]}$, or the accumulation of different "lines of evidence"; sample chemical analysis and bioanalysis, in situ functional responses, and field surveys ${ }^{[15]}$ may be highlighted, although the field remains open to new findings.

\section{Human health: wastewater-based epidemiology}

Pollution of water resources may pose a risk to human health through the drinking water supply, particularly in those areas where the potabilization treatment is poor or lacking. However, there is a second aspect of water pollution that has arisen as a powerful tool regarding human health. The so-called "wastewater-based epidemiology (WBE)", or "sewage epidemiology" ${ }^{[16]}$, estimates collective consumption or exposure to chemicals or pathogens by monitoring the substances excreted in the population's wastewater, thus providing an overall picture of the population health which is complementary to that obtained from clinical epidemiological surveys ${ }^{[17]}$. WBE was originally focused on small molecules, but the scope has since been enlarged to other biomarkers such as genetic biomarkers, large molecules, viruses, infection diseases, resistance genes, etc. Many successful studies using small molecules have been carried, out mostly on the chemical exposure side, namely, the consumption of illegal drugs, pharmaceuticals, and personal care products, tobacco and alcohol use, pesticide exposure, etc. Conversely, studies on the biological response side are less common. In this respect, the recent use of reverse transcription polymerase chain reaction and other related genomic methods for the monitoring of COVID-19 outbreak prevalence deserves to be mentioned ${ }^{[17-19]}$. Contrastingly, studies aimed at characterizing wastewater metabolomics and proteomics are lacking. Several biomarkers have been proposed for that purpose, notably protein biomarker ${ }^{[20]}$, but no systematic studies on wastewater proteomics had been published until very recently ${ }^{[21]}$. In that context, the possibility of using WBE mass spectrometry tools [LC-electrospray (ESI)-MS/MS and MALDI-TOF-MS] for characterization of SARS-CoV-2 proteins and peptides have been suggested, although the few examples analyzed reported for now in the literature correspond only to human clinical samples with none from wastewater ${ }^{[17,22]}$. 


\section{ANALYTICAL CHALLENGES}

The effects posed by chemical pollution on the water environment involve the quantification (measurement) of both exposure to chemicals and their effects on the biological receptors. Whereas exposure-oriented approaches tend to ignore data gaps (i.e., missing contaminants), effect-based approaches penalize them with increased uncertainty factors. For that reason, current monitoring approaches tend to emphasize either exposure or effect detection. However, it can be argued that their joint, complementary, and wise use paves the way to a better understanding of the causal chain connecting exposure and effects ${ }^{[15]}$.

\section{Chemical analysis}

Environmental samples contain a complex mixture of many constituents, including substances that can interfere with the analysis of emerging organic compounds. As mentioned, the advances achieved by highresolution mass spectrometry (HRMS), together with big data treatment tools (chemometric, bioinformatics, and databases) provide the suitable toolbox necessary to successfully cope with such a challenging analytical task ${ }^{[23]}$. MS target methods aimed at the quantification of certain groups of organic contaminants are often not sufficient to fully characterize such real mixtures. Up to a few hundred analytes can be simultaneously evaluated by current multi-residue targeted MS methods. Detection of unknowns has become more accessible with the advent of affordable, benchtop HRMS, among other tools, with improved selectivity, such as those based on time-of-flight (TOF) or Orbitrap-based mass spectrometry. In the standard target approach, once a particular compound is detected (i.e., its molecular peak $\mathrm{m} / \mathrm{z}$ ), a corresponding standard should be used to corroborate the identity and structure of the compound and to quantify it. As an alternative, the compound under consideration can be synthesized (in the case of TPs), or its identity can be confirmed using diverse spectral libraries. Structural elucidation of TPs is still one of the most employed procedures to assess the fate of emerging contaminants and is essential for the characterization of their transformation pathways. However, this is still a complex and challenging task requiring an array of instrumental techniques, such as gas chromatography-tandem MS (GC-MS) and liquid chromatography-tandem MS (LC-MS). A more comprehensive picture could be drawn using modern suspect and non-target analytical approaches to solve this challenge. The clear advantage of the non-target approach is the mining of the compounds present in the sample without resorting to direct but lengthy target analysis (provided that they are detectable). A drawback to consider is that the implementation of non-target approaches requires extensive use of data processing workflows (computational tools and software) to extract information from the massive amount of data generated by LC-MS in full-scan acquisition modes.

In summary, target analysis is limited to known analytes for which standards are available, thus allowing the development of multi-residue methods for their exact identification and quantification. In contrast, in suspect and non-target analysis, the number of analytes detected (and identified at different degrees of certitude) can be substantially broadened. However, since standards are not always available, they can only be semi-quantified. Moreover, suspect and non-target analysis strongly depend on compiled analytical information contained in external databases, and in the non-target analysis, even the structures of the compounds detected maybe are not necessarily previously known.

\section{Bioassays}

The number of bioassays and ecotoxicity tests available cover a vast range of possibilities, including in vivo vs. in vitro tests, apical effects (organisms) vs. specific effects (endocrine disruption, genotoxicity, mutagenicity, neurotoxicity, oxidative stress, etc.), acute (short-term) vs. chronic (long-term) exposures, different end-points, commercially available (Microtox ${ }^{\oplus}$, CALUX $^{\oplus}$, etc.) vs. research models, or even in silico methods for toxicity prediction for those compounds for which standards are not available ${ }^{[24]}$. There is a 
consensus on the convenience of using a battery of different bioassays covering different modes of action and endpoints to get a more comprehensive picture of the toxicity associated with a mixture of water pollutants.

Conventional ecotoxicological approaches typically make use of whole-organism in vivo bioassays to assess the toxicity of wastewater effluents. However, such bioassays exhibit some important drawbacks ${ }^{[25]}:(1)$ a limited sample throughput processing capability; (2) the inability to distinguish the effects of pollutants from those of the matrix (i.e., salinity, $\mathrm{pH}$, etc.); and (3) making unnecessary use of living organisms. This limitation was overcome with the development of cell-based in vitro bioassays with high-throughput processing capacity ${ }^{[26]}$. The application of such in vitro bioassays in ecological risk assessment is just at its beginnings but appears to be a promising tool for routine environmental surveys and reducing animal testing $^{[27]}$.

Considering that every constituent in a mixture can potentially contribute to its overall toxicity, the use of bioassays allows characterizing the joint effect of all the chemicals present. This can be contrasted with an estimation of the toxicity of the contaminants measured in the sample (known after chemical analysis) using aggregation models such as concentration addition or independent action ${ }^{[5,28]}$. The difference between measured and compound-based calculated toxicities would indicate that the chemical analysis is incomplete and further investigations are needed. This iterative process aiming to improve the explanation in the differences between measured and calculated toxicities drives the coupling between chemical and ecotoxicological monitoring and constitutes the underlying principle of the so-called effect-directed analysis ${ }^{[15,22]}$.

\section{BROADENING THE SCOPE OF WATER CONTAMINANTS: MICRO- AND NANO-PLASTICS}

Plastics have become essential materials in our modern society owing to their affordability and multitude of applications. This has led to an increase in the worldwide production to ca. 359 million tons in $2018^{[30]}$. Despite their undeniable advantages, the indiscriminate use of plastics as disposable material together with inadequate waste management has resulted in a source of environmental pollution of global concern. Noteworthy, the situation has substantially worsened as a consequence of the COVID-19 outbreak ${ }^{[3,31]}$. Land dumped plastic waste is transported by the river network ${ }^{[32]}$ with an annual estimated amount of 1.22.4 million tons in $2016^{[33]}$. Under business-as-usual scenarios, these emission rates could be doubled by $2025^{[34]}$. Plastic debris accumulates in the rivers, lakes, and the ocean (ranging from 9 to 23 million metric tons/year) ${ }^{[3,35]}$. Plastics are generally chemically stable; however, under weathering, they undergo fragmentation and give rise to microplastics $(<5 \mathrm{~mm}$ in any dimension) and nanoplastics $(<100 \mathrm{~nm})$, although this division is somewhat arbitrary. Such plastic particles are potentially harmful and may cause adverse effects on ecosystem biodiversity, changes in the carbon and nutrient cycles, habitat alterations, and harm human health ${ }^{[36,37]}$. However, the majority of the ecotoxicological studies carried out up to now are laboratory studies not using samples collected in the environment. On the other hand, plastics act as carriers of other emerging contaminants adsorbed in their surface ("Trojan effect"), thus facilitating their incorporation into the trophic chain. Even though plastics have already been ubiquitously reported in almost all of Earth's aquatic habitats, from the open seas to deep oceans, rivers, and lakes, either in the water column or the sediments, the environmental detection of MP/NP is still pending and remains an urgent research task to be undertaken in the forthcoming future ${ }^{[38]}$. In this context, Raman and Fourier-transform infrared spectroscopies (FT-IR and FT-Raman) and their microscopy versions, pyrolysis and thermal desorption gas chromatography coupled to mass spectrometry (Pyr-GC-MS and TED-GC-MS respectively), imaging techniques, or thermogravimetry coupled to differential scanning calorimetry deserve to be mentioned as the most prominent analytical methods and instrumentation for MP/NP characterization ${ }^{[38]}$. 


\section{Table 2. Subject areas of specific interest include (but are not limited to)}

- Studies of any aspect related to the occurrence, partition, transport, fate, and effects of the different families of emerging contaminants, such as pharmaceuticals, personal-care products, UV filters, pesticides, flame retardants, perfluorinated compounds and other industrial chemicals, disinfection by-products, persistent organic pollutants, endocrine disruptors, as well as their metabolites and environmental

transformation products

- Novel analytical methods, including new data treatment approaches (chemometric studies), suspect, and non-target screening methods

- Novel sampling methods (use of passive sampling devices, etc.)

- New emerging contaminants.

- Modeling studies on the occurrence, partition, transport, and fate of emerging contaminants in the aquatic environment (surface and groundwater), including the different involved phases and their validation using field data

- Studies about nanomaterials, nanocomposites, microplastics, and other related contaminants in the aquatic environment

- Studies (field, mesocosm, and microcosm) combining the occurrence and effects of emerging contaminants with other disciplines such as microbiology, ecology, genomics, hydrology, hydromorphology, etc.

- Studies related to the joint effects of water pollution by emerging contaminants and other environmental stressors on the receiving ecosystems

- Environmental determinants of both communicable and non-communicable water-borne diseases under the prism of climate change and its manifestations

- Risk assessment studies of emerging contaminants and their health and ecosystem risks

- Studies on contaminant (bio)monitoring and their environmental effects, such as the generation of resistance (antibiotic resistance genes, etc.)

- Studies on human and environmental biomonitoring of contaminants and their effects on individuals and populations, including the environment

- Metabolomic studies related to the response of aquatic living organisms to exposure to emerging contaminants

- Studies of Environmental Proteomics

- "One Health" or "exposome" approaches with emphasis on epidemiological studies about the combined effects of chemicals on human and ecosystem health

- Studies of advanced treatment methods used along the anthropogenic water cycle (i.e., drinking and wastewater treatment) for the elimination of emerging contaminants

- Studies related to new regulatory and policy aspects of broad concern related to the occurrence and effects of pollution by emerging contaminants and their mixtures in the water environment

With the analytical developments currently available, the environmental occurrence of MP/NPs can be conveniently accomplished (i.e., sampling, isolation, and characterization). However, there are some methodological needs concerning the analytical process still requiring further work, i.e., standardization and homogenization to enable the comparison of different studies, guidelines for methods validation, reference materials, or interlaboratory exercises ${ }^{[38]}$.

\section{CONCLUDING REMARKS: WHY A NEW JOURNAL ON EMERGING CONTAMINANTS AND NANOPLASTICS?}

Throughout the foregoing lines, we outline the most prominent areas of research within the field of water contaminants and nanoplastics. Indeed, it is a hot topic that has experienced tremendous growth, as reflected in the exponential number of scientific literature that appeared in the last 30 years [Figure 1]. It encompasses many areas of knowledge and research topics, which we aim to cover in our new journal "Water Emerging Contaminants \& Nanoplastics", as summarized in Table 2. We emphasize this multidisciplinary character since it is in the cross-boundary between different disciplines where new scientific ideas and approaches can flourish. In conclusion, we firmly believe that the journal comes at the right time and will definitely contribute to filling a scientific need. 
Emerging Contaminants in water
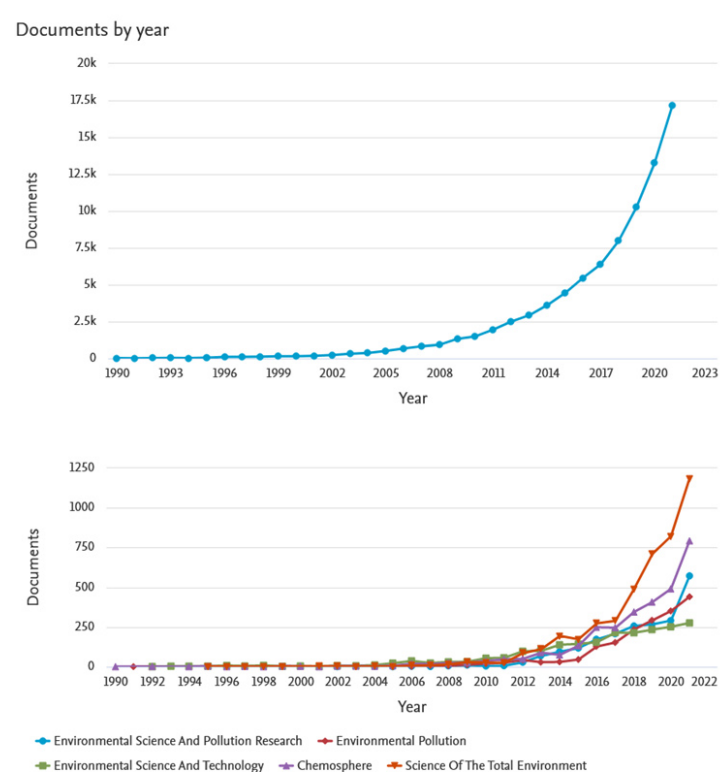

\section{Micro and Nanoplastics}
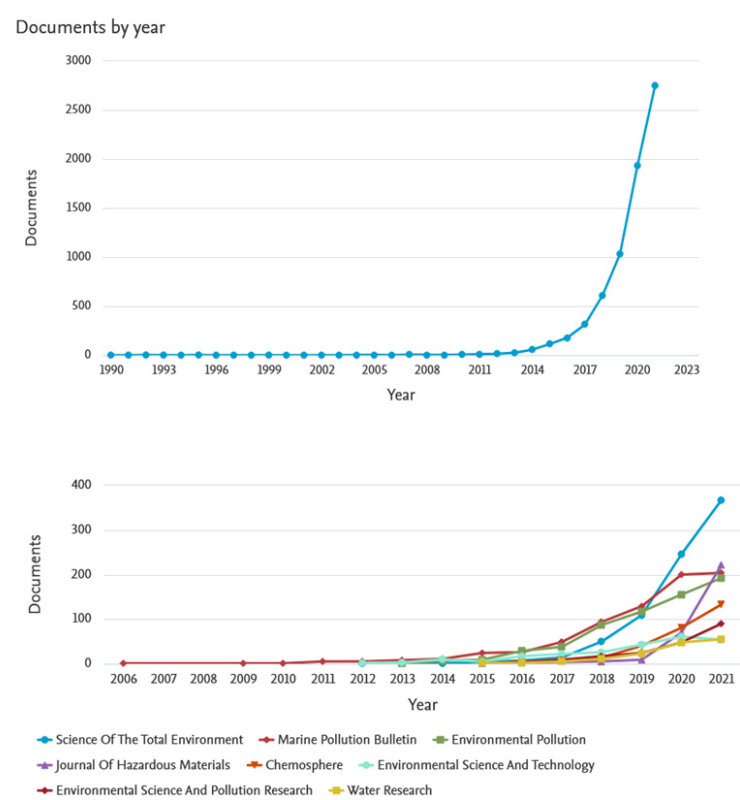

Figure 1. Evolution of the number of publications related to (left) "emerging contaminants in water" (right) and "micro- and nanoplastics" appearing in the last 30 years (1990-2021): (top) total aggregated number of publications and (bottom) number of publications in the main journals (source of data and graphs, SCOPUS; date downloaded, 29 November 2021).

\section{DECLARATIONS}

\section{Authors' contributions}

The author contributed solely to the article.

\section{Availability of data and materials}

Not applicable.

\section{Financial support and sponsorship}

None.

\section{Conflicts of interest}

The author declared that there are no conflicts of interest.

\section{Ethical approval and consent to participate}

Not applicable.

\section{Consent for publication}

Not applicable.

\section{Copyright}

(c) The Author(s) 2021.

\section{REFERENCES}

1. Kümmerer K, Clark JH, Zuin VG. Rethinking chemistry for a circular economy. Science 2020;367:369-70. DOI PubMed

2. Johnson AC, Jin X, Nakada N, Sumpter JP. Learning from the past and considering the future of chemicals in the environment. Science 2020;367:384-7. DOI PubMed

3. Kümmerer K. Sustainable chemistry: a future guiding principle. Angew Chem Int Ed Engl 2017;56:16420-1. DOI PubMed 
4. Kümmerer K, Dionysiou DD, Olsson O, Fatta-Kassinos D. A path to clean water. Science 2018;361:222-4. DOI PubMed

5. Kortenkamp A, Faust M. Regulate to reduce chemical mixture risk. Science 2018;361:224-6. DOI PubMed

6. Malaj E, von der Ohe PC, Grote M, et al. Organic chemicals jeopardize the health of freshwater ecosystems on the continental scale. Proc Natl Acad Sci U S A 2014;111:9549-54. DOI PubMed PMC

7. Posthuma L, de Zwart D, Dyer SD. Chemical mixtures affect freshwater species assemblages: from problems to solutions. Current Opinion in Environmental Science \& Health 2019;11:78-89. DOI

8. Groh KJ, Carvalho RN, Chipman JK, et al. Development and application of the adverse outcome pathway framework for understanding and predicting chronic toxicity: I. challenges and research needs in ecotoxicology. Chemosphere 2015;120:764-77. DOI PubMed

9. Edwards SW, Tan YM, Villeneuve DL, Meek ME, McQueen CA. Adverse outcome pathways-organizing toxicological information to improve decision making. J Pharmacol Exp Ther 2016;356:170-81. DOI PubMed

10. Vermeulen R, Schymanski EL, Barabási AL, Miller GW. The exposome and health: where chemistry meets biology. Science 2020;367:392-6. DOI PubMed PMC

11. Segner H, Schmitt-Jansen M, Sabater S. Assessing the impact of multiple stressors on aquatic biota: the receptor's side matters. Environ Sci Technol 2014;48:7690-6. DOI PubMed

12. Birk S, Chapman D, Carvalho L, et al. Impacts of multiple stressors on freshwater biota across spatial scales and ecosystems. Nat Ecol Evol 2020;4:1060-8. DOI PubMed

13. Sabater S, Barceló D, De Castro-Català N, et al. Shared effects of organic microcontaminants and environmental stressors on biofilms and invertebrates in impaired rivers. Environ Pollut 2016;210:303-14. DOI PubMed

14. Rico A, Van den Brink PJ, Leitner P, Graf W, Focks A. Relative influence of chemical and non-chemical stressors on invertebrate communities: a case study in the Danube River. Sci Total Environ 2016;571:1370-82. DOI PubMed

15. Altenburger R, Brack W, Burgess RM, et al. Future water quality monitoring: improving the balance between exposure and toxicity assessments of real-world pollutant mixtures. Environ Sci Eur 2019;31:12. DOI

16. Daughton CG. Monitoring wastewater for assessing community health: Sewage Chemical-Information Mining (SCIM). Sci Total Environ 2018;619-620:748-64. DOI PubMed PMC

17. Picó Y, Barceló D. Mass spectrometry in wastewater-based epidemiology for the determination of small and large molecules as biomarkers of exposure: toward a global view of environment and human health under the COVID-19 outbreak. ACS Omega 2021;6:30865-72. DOI PubMed PMC

18. Barceló D. Wastewater-Based Epidemiology to monitor COVID-19 outbreak: present and future diagnostic methods to be in your radar. Case Stud Chem Environ Eng 2020;2:100042. DOI

19. Alygizakis N, Markou AN, Rousis NI, et al. Analytical methodologies for the detection of SARS-CoV-2 in wastewater: protocols and future perspectives. Trends Analyt Chem 2021;134:116125. DOI PubMed PMC

20. Rice J, Kasprzyk-hordern B. A new paradigm in public health assessment: water fingerprinting for protein markers of public health using mass spectrometry. TrAC Trend Anal Chem 2019;119:115621. DOI

21. Carrascal M, Abian J, Ginebreda A, Barceló D. Discovery of large molecules as new biomarkers in wastewater using environmental proteomics and suitable polymer probes. Sci Total Environ 2020;747:141145. DOI PubMed

22. Griffin JH, Downard KM. Mass spectrometry analytical responses to the SARS-CoV2 coronavirus in review. Trends Analyt Chem 2021;142:116328. DOI PubMed PMC

23. Schymanski EL, Singer HP, Longrée P, et al. Strategies to characterize polar organic contamination in wastewater: exploring the capability of high resolution mass spectrometry. Environ Sci Technol 2014;48:1811-8. DOI PubMed

24. Barceló D, Žonja B, Ginebreda A. Toxicity tests in wastewater and drinking water treatment processes: a complementary assessment tool to be on your radar. J Environ Chem Eng 2020;8:104262. DOI

25. Escher BI, Stapleton HM, Schymanski EL. Tracking complex mixtures of chemicals in our changing environment. Science 2020;367:388-92. DOI PubMed PMC

26. Attene-Ramos MS, Miller N, Huang R, et al. The Tox21 robotic platform for the assessment of environmental chemicals--from vision to reality. Drug Discov Today 2013;18:716-23. DOI

27. Villeneuve DL, Coady K, Escher BI, et al. High-throughput screening and environmental risk assessment: State of the science and emerging applications. Environ Toxicol Chem 2019;38:12-26. DOI PubMed PMC

28. Backhaus T, Faust M. Predictive environmental risk assessment of chemical mixtures: a conceptual framework. Environ Sci Technol 2012;46:2564-73. DOI PubMed

29. Brack W, Ait-Aissa S, Burgess RM, et al. Effect-directed analysis supporting monitoring of aquatic environments--an in-depth overview. Sci Total Environ 2016;544:1073-118. DOI

30. Prata JC, Silva ALP, Walker TR, Duarte AC, Rocha-Santos T. COVID-19 pandemic repercussions on the use and management of plastics. Environ Sci Technol 2020;54:7760-5. DOI PubMed

31. Patrício Silva AL, Prata JC, Walker TR, et al. Increased plastic pollution due to COVID-19 pandemic: challenges and recommendations. Chem Eng J 2021;405:126683. DOI PubMed PMC

32. González-fernández D, Cózar A, Hanke G, et al. Floating macrolitter leaked from Europe into the ocean. Nat Sustain 2021;4:474-83. DOI

33. Lebreton LCM, van der Zwet J, Damsteeg JW, Slat B, Andrady A, Reisser J. River plastic emissions to the world's oceans. Nat Commun 2017;8:15611. DOI PubMed PMC

34. MacLeod M, Arp HPH, Tekman MB, Jahnke A. The global threat from plastic pollution. Science 2021;373:61-5. DOI PubMed 
35. Eriksen M, Lebreton LC, Carson HS, et al. Plastic pollution in the world's oceans: more than 5 trillion plastic pieces weighing over 250,000 tons afloat at sea. PLoS One 2014;9:e111913. DOI PubMed PMC

36. Rillig MC, Kim SW, Kim TY, Waldman WR. The global plastic toxicity debt. Environ Sci Technol 2021;55:2717-9. DOI PubMed PMC

37. Huerta Lwanga E, Mendoza Vega J, Ku Quej V, et al. Field evidence for transfer of plastic debris along a terrestrial food chain. Sci Rep 2017;7:14071. DOI PubMed PMC

38. Picó Y, Barceló D. Analysis and prevention of microplastics pollution in water: current perspectives and future directions. ACS Omega 2019;4:6709-19. DOI PubMed PMC 